\title{
Effects of Thermal Radiation on Hydromagnetic Flow due to a Porous Rotating Disk with Hall Effect
}

\author{
S.P. Anjali Devi ${ }^{1}$ and R. Uma Devi ${ }^{2 \dagger}$ \\ ${ }^{I}$ Department of Applied Mathematics, Bharathiar University, Coimbatore- 641 046, India \\ ${ }^{2}$ Department of Mathematics, Bharathiar University, Coimbatore- 641 046, India. \\ $\dagger$ Corresponding Author Email: r.umadevimaths@gmail.com
}

(Received October 15, 2009; accepted January 10, 2011)

\begin{abstract}
Radiation effect on steady laminar hydromagnetic flow of a viscous, Newtonian and electrically conducting fluid past a porous rotating infinite disk is studied taking Hall current into account. The system of axisymmetric nonlinear partial differential equations governing the MHD flow and heat transfer are reduced to nonlinear ordinary differential equations by introducing suitable similarity variables introduced by von Karman and the resulting nonlinear equations are solved numerically using Runge-Kutta based shooting method. A parametric study of all parameters involved was conducted and a representative set of results showing the effect of the magnetic field, the radiation parameter, the uniform suction/injection parameter and Hall parameter are illustrated graphically. The numerical values of the radial and tangential skin-friction coefficient and Nusselt number are calculated and displayed in the tables showing the effects of various parameters. Finally, a good comparison between the present numerical predictions and the previously published data are presented in the absence of magnetic field and radiation.
\end{abstract}

Keywords: Rotating disk, Hall effect, Radiation effect, Skinfriction coefficient, Nusselt number.

\section{NOMENCLATURE}

$\begin{array}{ll}C_{p} & \text { specific heat at constant pressure } \\ e & \text { charge of electron } \\ \vec{J} & \text { current density } \\ k & \text { thermal conductivity } \\ k^{*} & \text { mean absorpson coefficient } \\ M & \text { magnetic interaction parameter } \\ m & \text { Hall parameter } \\ P r & \text { prandtl number } \\ q_{r} & \text { radiative heat flux } \\ R & \text { radiation parameter } \\ T & \text { fluid temperature }\end{array}$

\section{INTRODUCTION}

Rotating disk flow along with heat transfer is one of the classical problems of fluid mechanics, which has both theoretical and practical value. The importance of heat transfer from a rotating body can be ascertained in cases of various types of machinery, for example computer disk drives and gas turbine rotors.

The first solution to the classical problem of rotating disk flow was obtained by von Karman (1921). His results were further improved by Cochran (1934). Benton (1966) and Rogar and Lanch (1960) extended this problem to the flow starting impulsively from rest. Considering the importance of suction/blowing, their

$\begin{array}{ll}u & \text { radial velocity } \\ v & \text { tangential velocity } \\ w & \text { axial velocity } \\ W_{S} & \text { suction/injection parameter } \\ \rho & \text { density of the fluid } \\ \sigma & \text { electrical conductivity } \\ \sigma^{*} & \text { Stefan Boltzmann constant } \\ \mu & \text { viscosity of the fluid } \\ v & \text { kinematic viscosity } \\ \Omega & \text { angular velocity } \\ (r, \phi, z) & \text { cylindrical polar coordinates }\end{array}$

effects on the surface of a rotating disk were studied by Stuart (1954), Kuiken, Sparrow et al. (1971). Suction essentially decreases both the radial and azimuthal components of velocity but increases the axial flow towards the disk at infinity. Abdul Maleque and Sattar (2005) investigated the steady laminar convective flow with variable properties due to a porous rotating disk. The problem still attracts the attention of researchers from various disciplines, since rotary type flow has many applications in different fields.

Magnetohydrodynamics which rises as a theory of the macroscopic interaction of electrically conducting fluids and electromagnetic fields has many practical 
applications in astronomy, space physics and geophysics as well as in many engineering fields. Some interesting results on the effects of the magnetic field on the steady flow due to the rotation of a disk of infinite or finite extent was pointed out by El-Mistikawy et al. (1990) and Attia and Hassan (2004).

In special, the study of hydromagnetic flows with Hall currents has important engineering application in problems of magnetohydrodynamics generators and of Hall accelerators as well as in flight magnetohydrodynamics. Hassan and Attia (1997) investigated the steady magnetohydrodynamics boundary layer flow due to an infinite disk rotating with uniform angular velocity in the presence of an axial magnetic field. They neglected induced magnetic field but considered Hall current and accordingly solved steady state equation numerically using finite difference approximation. The effect of Hall current on the fluid flow was studied by Hossain (1986) and Raptis and Ram (1984). Attia (1998) investigated the effects of suction as well as injection along with effects of magnetic field in a flow near a rotating disk. It was observed by him that strong injection tends to destabilize the laminar boundary layer but when magnetic field works along with even strong injection, it stabilizes the boundary layer.

Radiation effect on flow and heat transfer is important in the context of space technology and processes involving high temperature. The radiative flows of an electrically conducting fluid with high temperature in the presence of a magnetic field are encountered in electrical power generation, astrophysical flows, solar power technology, space vehicle re entry, nuclear engineering applications and other industrial areas. The thermal radiation of gray and electrically conducting fluid which is emitting and absorbing in a nonscattering medium has been examined by Ali et al. (1984), Ibrahim (1990), Raptis et al. (2004), Hossain et al. (1999) and Takhar et al. (1996). In the case of high temperature, radiation effects are quite significant. Recently, radiation and mass transfer effects on MHD free convective dissipative fluid in the presence of peat source/sink was analyzed by.

In the present paper, the steady hydromagnetic laminar flow with heat transfer of a viscous electrically conducting, incompressible flow due to the uniform rotation of a porous disk of infinite extent with an axial uniform steady magnetic field is analyzed in the presence of radiation effects. The uniform suction/ injection is applied through the surface of the disk. The temperature of the disk is maintained at a constant value. The governing non-linear partial differential equations are integrated numerically using Runge-Kutta based shooting method and utilizing Nachtsheim and Swigert (1965) iteration technique. The effect of radiation and Hall current are analysed in detail.

\section{Formulation of the Problem}

Effects of thermal radiation on steady hydromagnetic laminar flow due to an infinite porous rotating disk in an electrically conducting fluid with Hall Effect is studied. A non-rotating cylindrical polar coordinate system $(r, \varphi, z)$ where $z$ is the vertical axis in the cylindrical coordinate system with $r$ and $\varphi$ as the radial and tangential axes is chosen. The homogeneous, electrically conducting fluid occupies the region $\mathrm{z}>0$ with the rotating disk placed at $z=0$ and rotating with constant angular velocity $\Omega$ (see Fig. 1). The fluid velocity components are $(u, v, w)$ in the directions of increasing $(r, \varphi, z)$ respectively, the pressure is $P$, the density of the fluid $\rho$ and $T$ is the fluid temperature. The surface of the rotating disk is maintained at a uniform temperature $T_{w}$. Far away from the wall, the free stream is kept at a constant temperature $T_{\infty}$ and at constant pressure $P_{\infty}$. The fluid is assumed to be gray, emitting, and absorbing heat and the external uniform magnetic field is applied perpendicular to the surface of the disk and has a constant magnetic flux density $B_{0}$ which is assumed unchanging with a small magnetic Reynolds number $\left(R_{m}<<1\right)$. The magnetic Reynolds number is assumed to be small, so that the induced magnetic field is negligible. If the strength of the magnetic field is high, then one cannot neglect the Hall current. This is rather important case for some practical engineering problems.

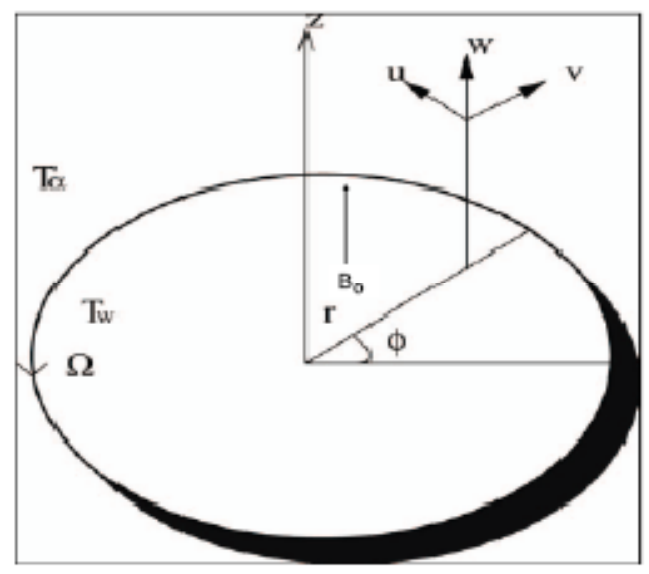

Fig. 1. Sketch of the problem

The governing equations of the fluid flow are given by Equation of Continuity:

$\nabla \cdot \overrightarrow{\mathrm{q}}=0$

Navier-Stokes equations:

$\rho(\vec{q} . \nabla) \vec{q}=-\nabla p+\mu \nabla^{2} \vec{q}+(\vec{J} \times \vec{B})$

The generalized Ohm's law:

$\vec{J}=\sigma[E+\vec{q} \times \vec{B}-\beta(\vec{J} \times \vec{B})]$

Energy Equation:

$\rho C_{P}(\vec{q} . \nabla) T=k \nabla^{2} T-\nabla q_{r}$

The external uniform magnetic field is applied perpendicular to the plane of the disk. Equation (3) expresses the Hall effect, where $\beta=\frac{1}{e n_{e}}$ is the Hall factor, $n e$ is the electron concentration per unit volume and $e$ is the charge of electron. Eq. (4) expresses the radiation effect, where $q_{r}=-\frac{4 \sigma^{*}}{3 k^{*}} \frac{\partial T^{4}}{\partial z}$ and the above set of equations takes the following form 
$\frac{\partial u}{\partial r}+\frac{u}{r}+\frac{\partial w}{\partial z}=0$

$\rho\left(u \frac{\partial u}{\partial r}-\frac{v^{2}}{r}+w \frac{\partial u}{\partial z}\right)+\frac{\partial p}{\partial r}=\mu \frac{\partial^{2} u}{\partial r^{2}}+\mu \frac{\partial}{\partial r}\left(\frac{u}{r}\right)+\mu \frac{\partial^{2} u}{\partial z^{2}}-$

$\frac{\sigma B_{0}{ }^{2}}{\left(1+m^{2}\right)}(u-m v)$

$\rho\left(u \frac{\partial v}{\partial r}+\frac{u v}{r}+w \frac{\partial v}{\partial z}\right)=\mu \frac{\partial^{2} v}{\partial r^{2}}+\mu \frac{\partial}{\partial r}\left(\frac{v}{r}\right)+\mu \frac{\partial^{2} v}{\partial z^{2}}-$

$\frac{\sigma B_{0}{ }^{2}}{\left(1+m^{2}\right)}(v+m u)$

$\rho\left(u \frac{\partial w}{\partial r}+w \frac{\partial w}{\partial z}\right)+\frac{\partial p}{\partial z}+\mu \frac{\partial}{\partial r}\left(\frac{\partial w}{\partial r}\right)+\frac{\mu}{r} \frac{\partial w}{\partial r}+\mu \frac{\partial}{\partial z}\left(\frac{\partial w}{\partial z}\right)$

$\rho C_{P}\left(u \frac{\partial T}{\partial r}+w \frac{\partial T}{\partial z}\right)=k \frac{\partial^{2} T}{\partial r^{2}}+\frac{k}{r} \frac{\partial T}{\partial r}+k \frac{\partial^{2} T}{\partial z^{2}}-\frac{\partial q_{r}}{\partial z}$

where $\sigma$ is the electrical conductivity, $C p$ is the specific heat at constant pressure, $q_{r}$ is the radiative heat flux and $m\left(=\sigma \beta B_{0}\right)$ is the Hall parameter.

Appropriate boundary conditions for the flow induced by an infinite disk $(z=0)$ which is started by impulsively into steady rotation with constant angular velocity $\Omega$ and a uniform suction/injection $w_{S}$ through the disk are given by

$\left\{u=0, v=\Omega r, w=w_{s}, T=T_{w}\right.$ at $z=0$

$\left\{u \rightarrow 0, v \rightarrow 0, T \rightarrow T_{\infty}, P \rightarrow P_{\infty}\right.$ as $z \rightarrow 0$

By using the Rosseland approximation for radiation for an optically thick layer, one can write

$q_{r}=-\frac{4 \sigma^{*}}{3 k^{*}} \frac{\partial T^{4}}{\partial z}$

where $\sigma^{*}$ is the Stefan-Boltzmann constant and $k^{*}$ is the mean absorption coefficient.

It is assumed that the temperature differences within the flow are such that the term $T^{4}$ may be expressed as a linear function of temperature. This is accomplished by expanding $T^{4}$ in a Tailor series about $T_{\infty}$ and neglecting higher order terms, we get

$T^{4} \cong 4 T_{\infty}{ }^{3} T-3 T_{\infty}{ }^{4}$

In view of Eqs. (11) and (12), Eq. (9) reduces to

$\rho C_{P}\left(u \frac{\partial T}{\partial r}+w \frac{\partial T}{\partial z}\right)=k \frac{\partial^{2} T}{\partial r^{2}}+\frac{k}{r} \frac{\partial T}{\partial r}+k \frac{\partial^{2} T}{\partial z^{2}}+\frac{16 \sigma^{*} T_{\infty}{ }^{3}}{3 k^{*}} \frac{\partial^{2} T}{\partial z^{2}}$

\section{SIMILARITY TRANSFORMATION}

To obtain the solutions of the governing equations, following von Karmann, a dimensionless normal distance from the disk, $\eta=z(\Omega / v)^{\frac{1}{2}}$ is introduced along with the following representations for the radial, tangential and axial velocities, pressure and temperature distributions:

$\left\{\begin{array}{c}u=\Omega r F(\eta), v=\Omega r G(\eta) \quad w=(\Omega v)^{\frac{1}{2}} H(\eta) \\ P-P_{\infty}=2 \mu \Omega p(\eta) \text { and } T-T_{\infty}=\Delta T \Theta(\eta)\end{array}\right.$ where $v$ is a uniform kinematic viscosity of the fluid and $\Delta T=T_{w}-T_{\infty}$. Equations (5), (7) and (9) in this case reduce to the system

$$
\begin{aligned}
& 2 F+H^{\prime}=0 \\
& F^{\prime \prime}-H F^{\prime}-F^{2}+G^{2}-\frac{M}{\left(1+m^{2}\right)}(F-m G)=0 \\
& G^{\prime \prime}+H G^{\prime}-2 F G-\frac{M}{\left(1+m^{2}\right)}(G+m F)=0 \\
& \text { And } \\
& \Theta-P r H \Theta^{\prime}-\frac{4}{3} \frac{1}{R} \Theta=0
\end{aligned}
$$

where $M=\frac{\sigma B_{0}}{\Omega \rho}$ is the magnetic parameter, $\operatorname{Pr}=\frac{\mu C_{P}}{k}$ is the Prandtl number and $\frac{k^{*} k}{4 \sigma^{*}} T_{\infty}{ }^{3}$ is the radiation parameter. The boundary conditions (10) transform to

$\left\{\begin{array}{c}F(0)=0, G(0)=1, H(0)=W_{s}, \Theta(0)=1 \\ F(\infty)=G(\infty)=\Theta(\infty)=p(\infty)=0\end{array}\right.$

where $W_{S}=w_{S} / \sqrt{v \Omega}$ represents a uniform suction when $W s<0$ and injection when $W s>0$ at the surface. The boundary conditions (19) imply that both the radial velocity $(F)$, the tangential velocity $(G)$, temperature and pressure vanish sufficiently far away from the rotating disk, whereas the axial velocity component $(H)$ is anticipated to approach a yet unknown asymptotic limit for sufficiently large $\eta$-values.

The skin friction coefficients and the Nusselt number are given by the Newtonian formulas:

$\tau_{t}=\left[\mu\left(\frac{\partial v}{\partial z}+\frac{1}{r} \frac{\partial w}{\partial \varphi}\right)\right]_{z=0}=\mu G^{\prime} \Omega(R e)^{\frac{1}{2}}$

$\tau_{t}=\left[\mu\left(\frac{\partial u}{\partial z}+\frac{\partial w}{\partial r}\right)\right]_{z=0}=\mu F^{\prime} \Omega(R e)^{\frac{1}{2}}$

Hence the tangential and radial skin-frictions are respectively given by

$(R e)^{\frac{1}{2}} C_{f_{t}}=G^{\prime}(0)$

$(R e)^{\frac{1}{2}} C_{f_{r}}=F^{\prime}(0)$

Rate of heat transfer is given by

$q=-\left(k \frac{\partial t}{\partial z}\right)_{z=0}=-k \Delta T\left(\frac{\Omega}{v}\right)^{\frac{1}{2}} \Theta^{\prime}(0)$

Hence the Nusselt number $(\mathrm{Nu})$ is obtained as

$(R e)^{-\frac{1}{2}} N u=-\Theta^{\prime}(0)$

where $\operatorname{Re}\left(=\Omega r^{2} / \mathrm{v}\right)$ is the rotational Reynolds number.

\section{Method of Solution}

Equations (15)-(18) are highly nonlinear and cumbersome to solve. Hence they are solved numerically using RK-Fourth order based shooting method with the utilization of Natchtsheim and Swigirt iteration scheme for satisfaction of the asymptotic boundary conditions, for different values of the physical parameters Magnetic interaction parameter $(M)$, Radiation parameter $(R)$, Suction/injection parameter $(W s)$ and Hall parameter $(m)$. 


\section{RESULTS AND DISCUSSION}

As a result of the numerical calculations, the velocity and temperature distributions for the flow are obtained from Eqs. (15)-(18) and are displayed in Figs. 2-5 for different values of $M$ (magnetic parameter), $m$ (hall parameter) $R$ (radiation parameter) and $W s$ (suction/injection parameter). In the present analysis the fluid considered is air. For air $(P r=0.71)$. We have confined our analysis to the case when we have suction and injection velocities that is when $(W s)<0$ and $(W s)$ $>0$.

Table 1 Numerical values of the radial and tangential skin friction coefficients and the rate of heat transfer coefficient for $\operatorname{Pr}=0.71$ and $M=m=0$ and $1 / R \rightarrow 0$

\begin{tabular}{|c|c|c|c|}
\hline$W_{s}$ & \multicolumn{3}{|c|}{ Present } \\
\hline & $F^{\prime}(0)$ & $-G(0)$ & $-\theta^{\prime}(0)$ \\
\hline 4 & 0.243043 & $0.289210 \mathrm{e}-1$ & $0.150101 \mathrm{e}-4$ \\
\hline 3 & 0.309185 & $0.602989 \mathrm{e}-1$ & $0.126771 \mathrm{e}-2$ \\
\hline 2 & 0.398941 & 0.135959 & $0.116090 \mathrm{e}-1$ \\
\hline 1 & 0.489481 & 0.302173 & $0.850176 \mathrm{e}-1$ \\
\hline 0 & 0.510214 & 0.615926 & 0.326831 \\
\hline-1 & 0.389564 & 1.175221 & 0.793069 \\
\hline-2 & 0.242416 & 2.038527 & 1.437784 \\
\hline-3 & 0.165576 & 3.012142 & 2.135586 \\
\hline-4 & 0.124738 & 4.220518 & 2.842382 \\
\hline$W_{s}$ & \multicolumn{2}{|c|}{ Kelson and Desseaux[(12)] } \\
\hline & $F^{\prime}(0)$ & $-G(0)$ & $-\theta^{\prime}(0)$ \\
\hline 4 & 0.243044 & $0.289211 \mathrm{e}-1$ & $0.107326 \mathrm{e}-4$ \\
\hline 3 & 0.309147 & $0.602893 \mathrm{e}-1$ & $0.576744 \mathrm{e}-3$ \\
\hline 2 & 0.398934 & 0.135952 & $0.110135 \mathrm{e}-1$ \\
\hline 1 & 0.489481 & 0.302173 & $0.848848 \mathrm{e}-1$ \\
\hline 0 & 0.510233 & 0.615922 & 0.325856 \\
\hline-1 & 0.389569 & 1.175222 & 0.793048 \\
\hline-2 & 0.242421 & 2.038527 & 1.437782 \\
\hline-3 & 0.165582 & 3.012142 & 2.135585 \\
\hline-4 & 0.124742 & 4.005180 & 2.842381 \\
\hline
\end{tabular}

In order to highlight the validity of the numerical computations adopted in the present investigation, some of our results have been compared with those of Kelson and Desseaux (1971) when there is no magnetic field and radiation parameter which are displayed through Table 1. The comparisons show excellent agreement, hence an encouragement for the use of the present numerical computations.

Figures $2 a-2 d$ show the effect of the magnetic field on velocity and temperature profiles. Radial, tangential and axial velocity decelerate as $M$ increases as shown in Figs. 2a, 2b and 2c. A drag like force called Lorentz force is generally created due to the introduction of the magnetic field which has the tendency to decelerate the flow around the disk at the expense of increasing its temperature.

Figures $3 \mathrm{a}, 3 \mathrm{~b}, 3 \mathrm{c}$ and $3 \mathrm{~d}$ show the $F, G,-H$ and $\theta$ profiles at different values of $m$. From Fig. 3b we observe that the Hall parameter $m$ has slightly increasing effect on the tangential velocity. Unlike the tangential velocity, the parameter $m$ has a marked effect on radial and axial velocity profiles as seen in Figs. 3a and $3 \mathrm{c}$. It is observed that, due to an increase in the values of $m$ within 0.0 and 2.0, both the radial and axial velocity increase. But if the magnitude of $m$ is increased beyond the limit of 2.0, the velocity profiles show a decreasing effect. This is due to the fact that for large value of $m$, the term $1 /\left(1+m^{2}\right)$ is very small and hence the resistive effect of the magnetic field is diminished.

The Hall current parameter $m$ and magnetic interaction parameter $M$ do not enter directly into the energy Eq. (19) but its influence come through the momentum Eqs. (16) and (17). Figure 2d and Fig. 3d show the variation of temperature distribution $\theta$ for different values of $M$ and $m$ respectively. From Fig. 2 d, it is observed that temperature increases with the increasing value of $M$. However it is insignificant. The temperature decreases with the increasing value of Hall parameter $m$ which is shown through Fig. $3 d$.

The effects of thermal radiation parameter $R$ for $M=$ 1.0, $m=0.5, W s=-1.0$ and $P r=0.71$ on the temperature is shown in Fig. $4 \mathrm{a}$ and it is seen that the radiation parameter $(R)$ caused reductions in the fluid temperature $\theta$. The Nusselt number increases with the increasing values of $R$ which is shown in Fig. 4b. The effects of suction and injection parameter $(W s)$ for $M=$ $1.0, m=0.5, R=1.0$ and $P r=0.71$ on the radial, tangential, axial velocity and temperature are shown in Figs. 5a-5d. From these figures, it is seen that the effect of suction is to decrease the radial, tangential and axial velocity components and temperature whereas the effect of injection is to increase the radial, tangential and axial velocities and temperature distribution.

Numerical values for skin friction coefficients, Nusselt number are obtained for various values of $M, m, R$ and $W s$ and they are displayed through Table 2.

Table 2 Variation of $F^{\prime}(0),-G^{\prime}(0)$ and $-\theta^{\prime}(0)$ at the disk surface with $M, m, R$ and $W s$.

\begin{tabular}{|rrrr|c|c|c|}
\hline$M$ & $m$ & $R$ & $\mathrm{~W} s$ & $F(0)$ & $-G^{\prime}(0)$ & $-\theta^{\prime}(0)$ \\
\hline 0.0 & 0.5 & 1.0 & -1.0 & 0.3916 & 1.1779 & 0.3772 \\
0.5 & 0.5 & 1.0 & -1.0 & 0.4072 & 1.4325 & 0.3724 \\
1.0 & 0.5 & 1.0 & -1.0 & 0.4299 & 1.6342 & 0.3700 \\
1.5 & 0.5 & 1.0 & -1.0 & 0.4538 & 1.8061 & 0.3682 \\
2.0 & 0.5 & 1.0 & -1.0 & 0.4774 & 1.9590 & 0.3668 \\
\hline 1.0 & 0.0 & 1.0 & -1.0 & 0.2510 & 1.6570 & 0.3496 \\
1.0 & 1.0 & 1.0 & -1.0 & 0.5126 & 1.5392 & 0.3818 \\
1.0 & 2.0 & 1.0 & -1.0 & 0.5298 & 1.3973 & 0.3891 \\
1.0 & 5.0 & 1.0 & -1.0 & 0.4771 & 1.2657 & 0.3876 \\
1.0 & 10 & 1.0 & -1.0 & 0.4400 & 1.2198 & 0.3837 \\
\hline 1.0 & 0.5 & 1.0 & -1.0 & 0.4299 & 1.6343 & 0.3700 \\
1.0 & 0.5 & 3.0 & -1.0 & 0.4299 & 1.6343 & 0.5590 \\
1.0 & 0.5 & 5.0 & -1.0 & 0.4299 & 1.6343 & 0.6306 \\
1.0 & 0.5 & 10 & -1.0 & 0.4299 & 1.6343 & 0.6992 \\
1.0 & 0.5 & 20 & -1.0 & 0.4299 & 1.6343 & 0.7400 \\
\hline 1.0 & 0.5 & 1.0 & 2.0 & 0.4324 & 0.4442 & 0.0371 \\
1.0 & 0.5 & 1.0 & 1.0 & 0.4894 & 0.6805 & 0.1003 \\
1.0 & 0.5 & 1.0 & 0.0 & 0.4952 & 1.0626 & 0.1758 \\
1.0 & 0.5 & 1.0 & -1.0 & 0.4299 & 1.6343 & 0.3692 \\
1.0 & 0.5 & 1.0 & -2.0 & 0.3360 & 2.3933 & 0.6368 \\
\hline
\end{tabular}



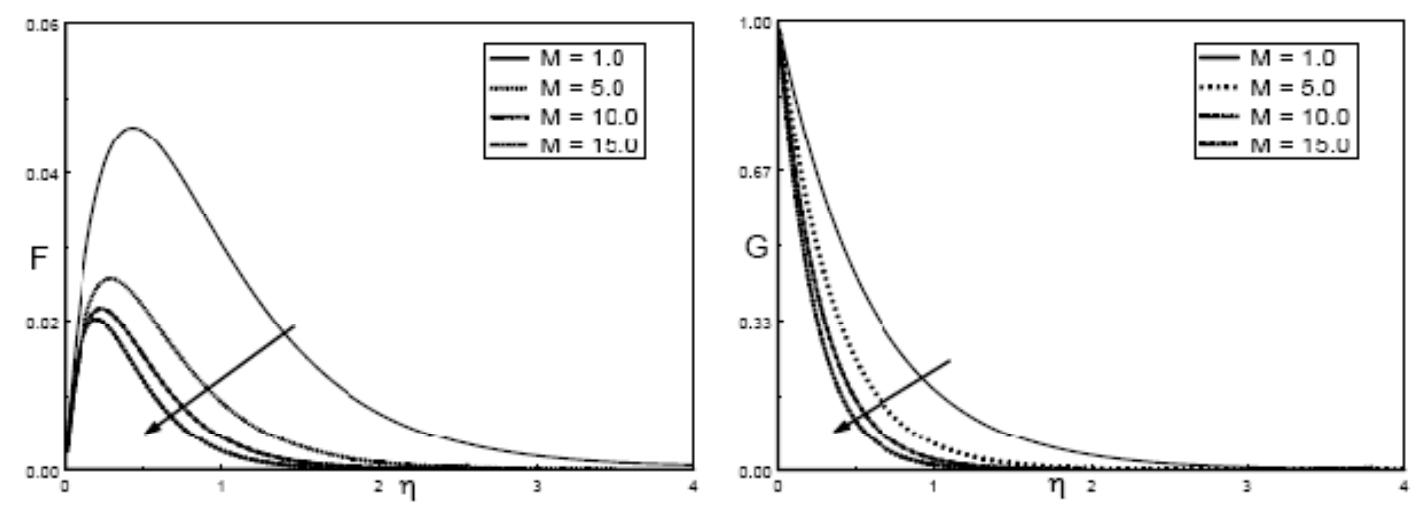

1(a)
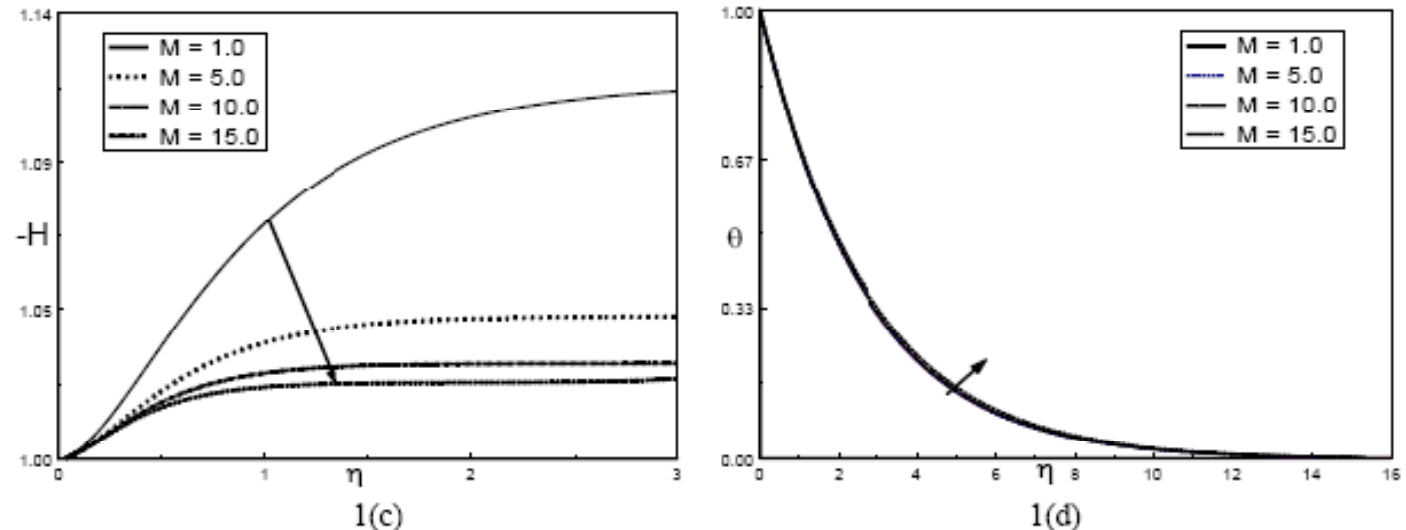

Fig. 2.Variation of radial, tangential and axial velocity profile and temperature distribution for different values of $M$ and for $m=0.5, W=-1.0, R=1.0 \& \operatorname{Pr}=0.71$.
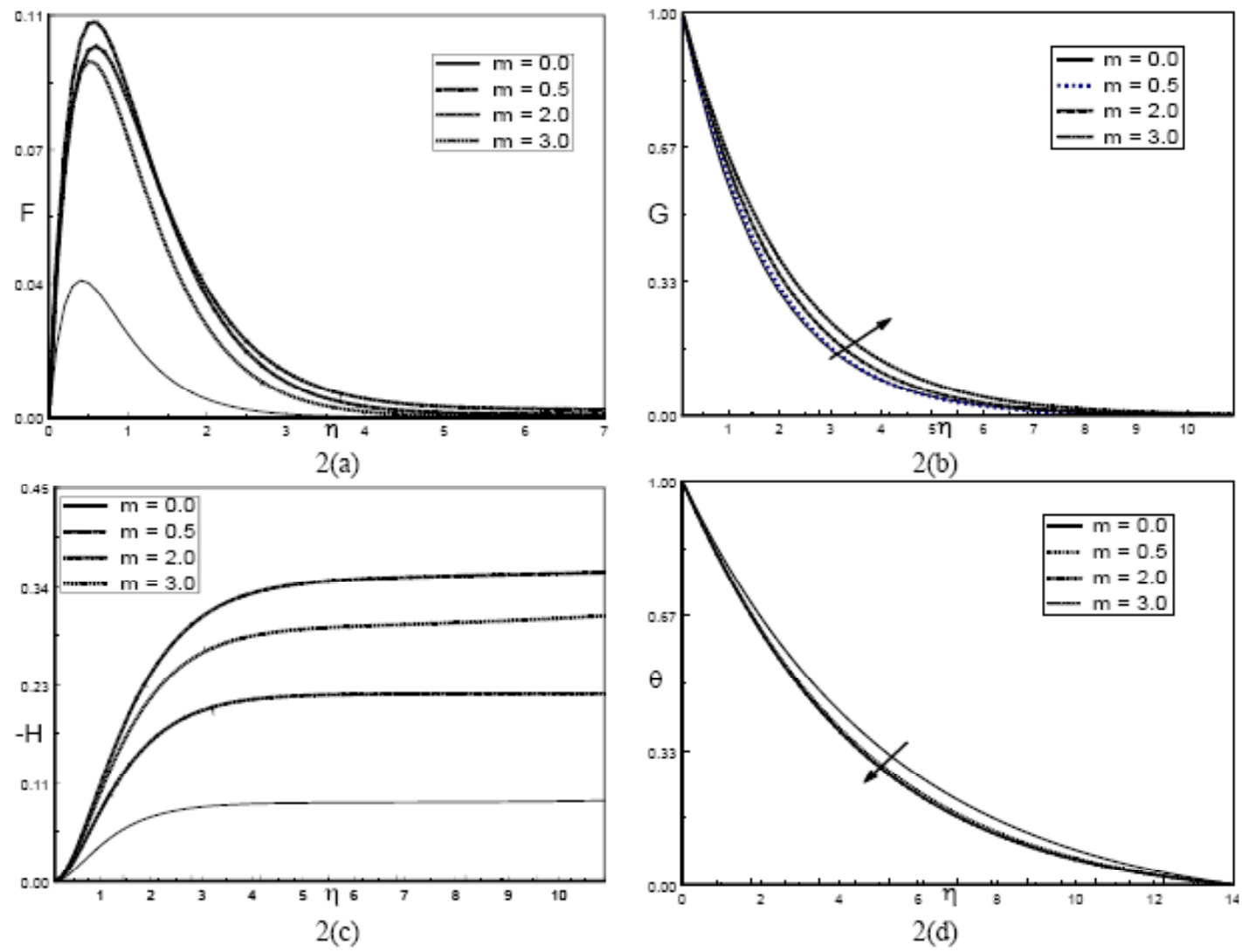

Fig. 3.Variation of radial, tangential and axial velocity profiles and temperature distribution for different values of $m$ and for $M=1.0, W=-1.0, R=1.0 \& P r=0.71$. 

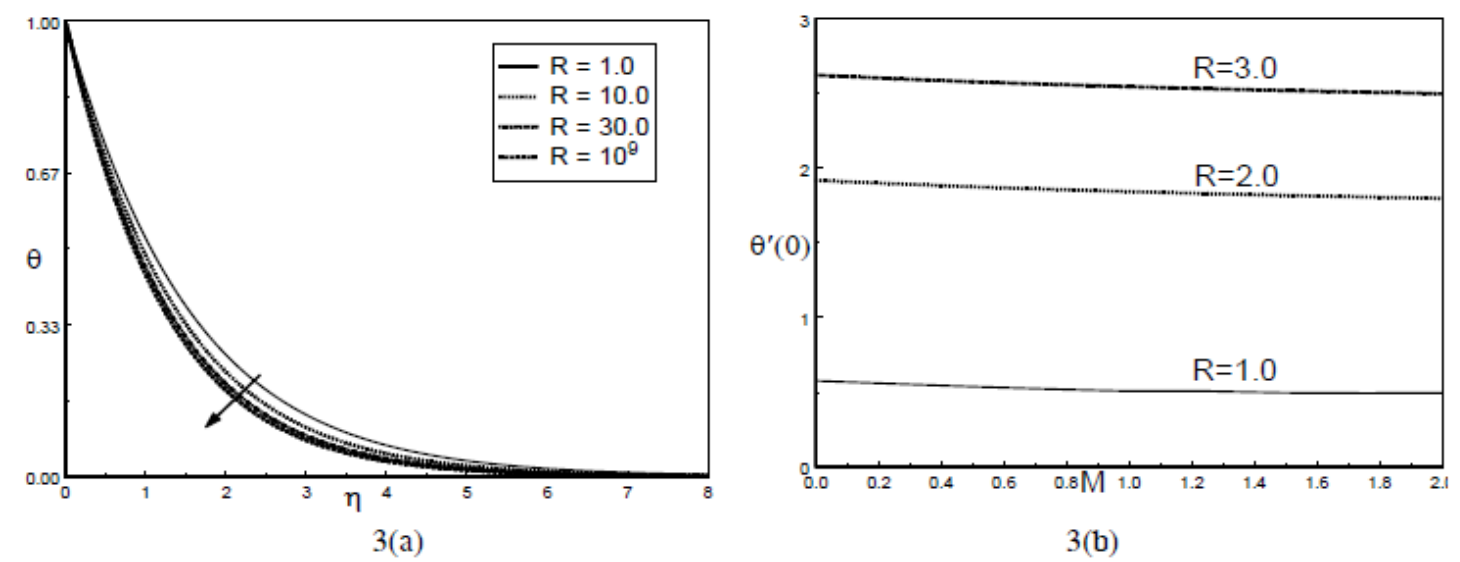

Fig. 4. (a) Variation of temperature distribution for different values of $R$ and for $W=-1.0, m=0.5, M=1.0$ \& $\operatorname{Pr}=0.71$. (b)Variation of skin friction profile for $\mathrm{R}=1,2 \& 3$
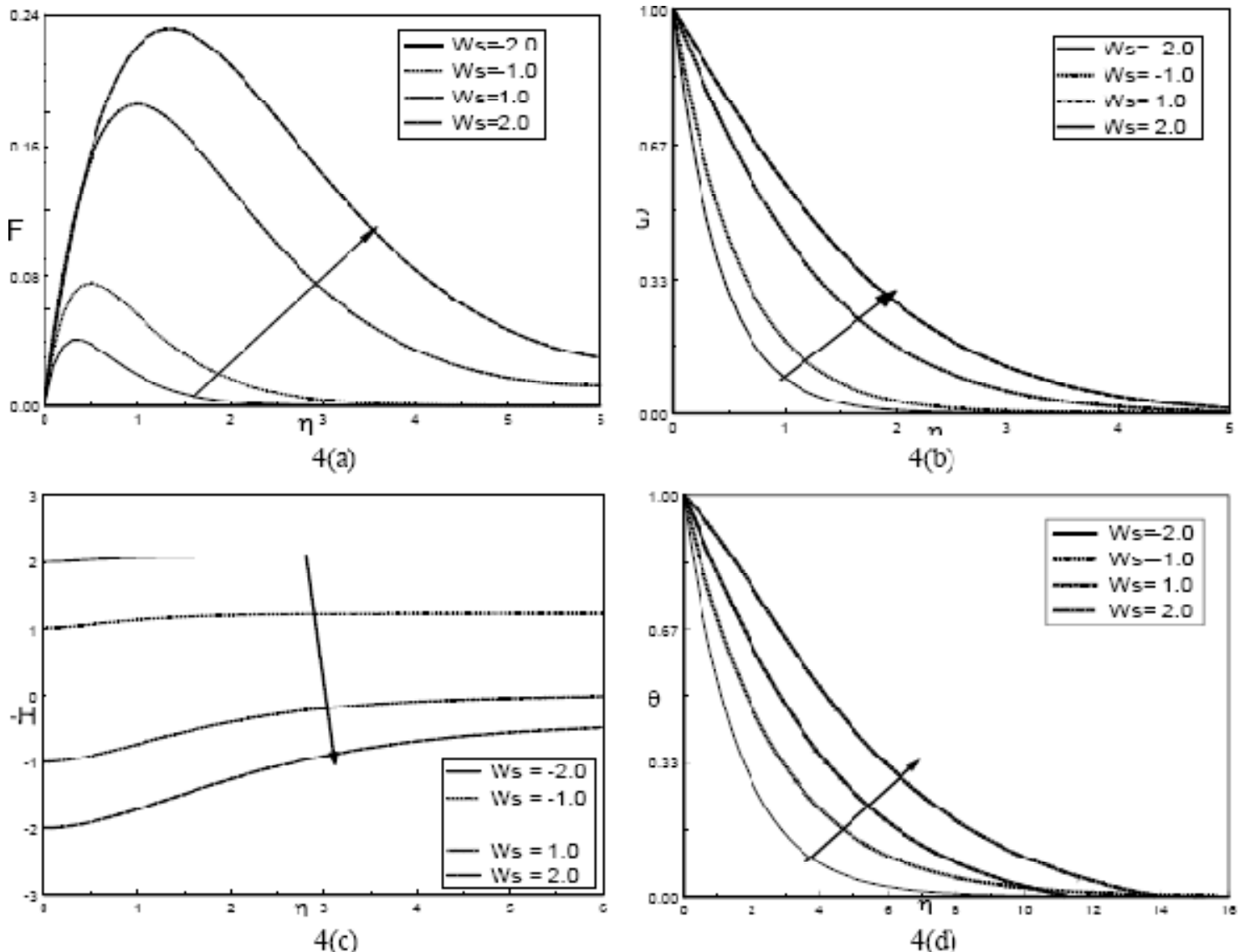

Fig. 5.Variation of radial, tangential and axial velocity profiles and temperature distribution for different values of $W s$ and for $m=0.5, M=1.0, R=1.0 \& \operatorname{Pr}=0.71$.

From Table 2, it is seen that the increase in $M$ lead to increase in radial and tangential skin frictions respectively whereas its influence is to decrease the rate of heat transfer. The effect of $m$ has increasing trend over radial skin-friction for $0 \leq m \leq 2.0$ where as it has a decreasing for $m \geq 2$. The tangential skin-friction increases with an increasing value of the Hall parameter $m$. It can be seen that the Nusselt number increases with an increasing value of $R$. It is noted that the values of the radial and tangential skin friction and the rate of heat transfer coefficients decrease for increasing values of injection velocity. It is also noted from this table that increasing the suction velocity leads to decrease in the radial skin friction coefficient and the tangential skinfriction coefficient while its effect is to increase the rate of heat transfer coefficient.

\section{Conclusion}

In this paper, the effect of thermal radiation on steady laminar convective hydrodynamic flow of an electrically conducting fluid over a porous rotating infinite disk was studied taking hall current into account. The Nachtsheim and Swigert [20] iteration technique based on fourth-order Range-Kutta and Shooting method has been employed to complete the 
integration of the resulting solutions. In the absence of radiation and magnetic field our results reduces to that of Kelson and Desseaux (1971).

The following conclusions are drawn as a result of the computations:

- Increasing values of $M$ lead to decrease in radial, tangential, axial velocities while it lead to increases in the temperature distribution.

- Hall parameter $m$ has an interesting effect on the radial and axial velocities so as to increasing values for $0 \leq m \leq 2.0$ where as it decreases them for $m \geq 2$. When $m$ decreases, the tangential and temperature reduce.

- The effect of the suction parameter is to decelerate the radial, tangential, axial velocities and temperature distribution while it has the opposite trend for increasing injection values.

- The influence of radiation parameter caused reduction in the fluid temperature and to increase the Nusselt number.

\section{REFERENCES}

Abdul Maleque, Kh. and Md. Abdul Sattar (2005). Steady laminar Convective flow with variable properties due to a porous rotating disk. J. of Heat Transfer 127, 1406-1409.

Ali, M.M., T.S. Chen and B.F. Armaly (1984). Natural convection-radiation interaction in boundary layer flow over horizontal surface. AIAA Journal 22, 1797-1803.

Attia, H.A. (1998). Unsteady MHD flow near a rotating porous disk with uniform suction or injection. Fluid Dyn. Res. 23, 283-290.

Attia, H.A. and A.L. Aboul-Hassan (2004). On hydromagnetic flow due to a rotating disk. Appl. Math. Modelling 28, 1007-1014.

Benton, E.R. (1966). On the flow due to a rotating disk. J. Fluid Mech. 24, 781-800.

Cochran, W.G. (1934). The flow due to a rotating disk. Proc. Camb. Philos. Soc. 30, 365-375.

El-Mistikawy, T.M.A., H.A. Attia and A.A. Megahed (1990, November). The rotating disk flow in the presence of a weak magnetic field. In Proc. 4th Conference on Theoretical and Appl. Mech., Cairo, Egypt, 69-82.

Hassan, A.L.A. and Hazem Ali Attia (1997). Flow due to a rotating disk with Hall effect. Phys. ltts. A 228, 246-290.

Hossain, M.A., M.A. Alim and D. Rees (1999). The effect of radiation on free convection from porous vertical plate. Int. J. Heat and Mass Transfer 42, 181-191.

Hossain, M.A. (1986). Effect of Hall Current on unsteady hydromagnetic free convection flow near an infinite vertical porous plate. J. Phys. Soc. Japan 55, 2183-2190.

Ibrahim, F.S. (1990). Mixed convectionradiation interaction in boundary-layer flow over horizontal surfaces. Ast. Phys. and Space Sci. 68, 263-276.

Kelson, N., A. Desseaux (1971). Note on porous rotating disk flow. ANZIAM J. 42 ,C847- C855.

Kuiken, H.K. (1971). The effect of normal blowing on the flow near a rotating disk of infinite extent. $J$. Fluid Mech. 47, 789-798.

Nachtsheim, P.R. and P. Swigert (1965). Satisfaction of Asymptotic Boundary conditions in Numerical Solution of System of Nonlinear Boundary Layer type. NASA TND3004.

Raptis, A., C. Perdikis and H.S. Takhar (2004). Effect of thermal radiation on MHD flow. Appl. Math. and comp.153, 645-649.

Raptis, A. and P.C. Ram (1984). Effects of Hall Current and rotation. Astrophys. Space Sci. 106, 257-264.

Rogers, M.G. and G.N. Lance (1960). The rotationally symmetric flow of a viscous fluid in presence of infinite rotating disk. J.Fluid Mech. 7, 617-631.

Sparrow, E.M., G.S. Beavers and L.Y. Hung (1971). Flow about a porous-surfaced rotating disk. Int. J. Heat and Mass Transfer 14, 993-996.

Stuart, J.T. (1954). On the effect of uniform suction on the steady flow due to a rotating disk. Q.J. Mech. Appl. Math. 7, 446-457.

Suneetha, S., N.B. Reddy and V.R. Prasad (2011). Radiation and mass Transfer effects on MHD free convective dissipative fluid in the presence of heat source/sink. J. Appl. Fluid Mech. 4, 107-113.

Takhar, H.S., R. Gorla and V.M. Soundalgekar (1996). Radiation effects on MHD free convection flow of gas past a semi-infinite vertical plate. Int. J. of Numerical Methods Heat and Fluid Flow 6, 77-83.

Von Karman, T. (1921). Uber laminar and turbulent Reibung, Z. Angew. Math. Mech. 1, 233-255. 